phys. stat. sol. (a) 139, 241 (1993)

Subject classification: $78.65 ; 66.30 ;$ S11.1

Fachbereich Physik (a) and Institut für Chemie (b) der Universitüt Osnabrück ${ }^{1}$ )

\title{
Concentration and Refractive Index Profiles of Titanium- and Iron-Diffused Planar $\mathrm{LiNbO}_{3}$ Waveguides
}

\author{
By \\ D. KiP (a), B. Gather (b), H. Bendig (b), and E. Krätzig (a)
}

\begin{abstract}
Depth profiles of titanium, iron, and niobium concentrations are investigated with an electron microprobe in planar optical $\mathrm{LiNbO}_{3}: \mathrm{Ti}$ waveguides additionally doped with iron. The profiles are well described by Gaussian functions allowing the determination of diffusion constants. The extraordinary refractive index of the waveguides can be described by a linear superposition of iron and titanium profiles.
\end{abstract}

\section{Introduction}

$\mathrm{LiNbO}_{3}$ is a promising material for applications in holographic volume storage and integrated optics [1]. Optical waveguides fabricated in single domain crystals of this material by titanium in-diffusion or proton exchange are used as integrated switches, modulators, or spectrometers $[2,3]$. Photorefractive iron- and copper-doped $\mathrm{LiNbO}_{3}$ waveguides enable efficient two- and four-wave mixing [4]. In particular, phase-conjugate waves can be generated by anisotropic four-wave mixing in a waveguide with very large efficiencies [5].

In this paper we report on the fundamental properties of $\mathrm{LiNbO}_{3}: \mathrm{Ti}$ waveguides additionally doped with iron $\left(\mathrm{LiNbO}_{3}: \mathrm{Ti}: \mathrm{Fe}\right)$. Photorefractive properties of the waveguides can be improved by high-temperature diffusion of transition metals, e.g. iron, from the surface. This process, however, leads to a strong change of the refractive index profiles of the waveguide, too. We analyse the dependence of the refractive index change on the concentration of in-diffused iron and determine the diffusion constants of titanium and iron. The knowledge of the profiles of both titanium and iron permits the investigation of depth-dependent photorefractive properties of the waveguides, e.g. conductivity or photovoltaic constants [4].

\section{Experimental Methods}

Our waveguides are prepared in two steps using $y$-cut $\mathrm{LiNbO}_{3}$ wafers of congruently melting composition. At first a thin titanium layer with a thickness of some tens of $\mu \mathrm{m}$ is vacuum-deposited on the $\mathrm{LiNbO}_{3}$ substrate and then annealed at a temperature of $1000^{\circ} \mathrm{C}$ in an atmosphere of wet argon gas. The addition of water prevents the out-diffusion of lithium from the surface of the substrate. In a second step, a thin iron layer is in-diffused in the same manner as the titanium layer. Here we use an atmosphere of wet oxygen, and thus the iron ions are oxidized to $\mathrm{Fe}^{3+}$ during annealing.

1) Barbarastr. 7, D-49069 Osnabrück, Federal Republic of Germany. 
The effective refractive indices of the TE and TM modes of the waveguides are measured by the prism coupling method (dark line spectroscopy). From the effective refractive indices the profiles of ordinary and extraordinary refractive index are reconstructed by the use of an inverse WKB method [6].

For the measurement of the concentration profiles the rear face of the planar $\mathrm{LiNbO}_{3}$ waveguides is polished. This face and the surface of the waveguide form an angle of $(90 \pm 1)^{c}$. The electron beam of a microprobe (acceleration voltage $15 \mathrm{keV}$, beam width $0.2 \mu \mathrm{m}$, step width $0.2 \mu \mathrm{m}$ ) scans the polished rear face starting from the surface. In two successive measurements the niobium and titanium and the niobium and iron concentrations are determined, respectively. Because of the low atomic number of lithium $(Z=3)$, this element is not detectable by this method. The niobium signal is used as reference which allows to defect the surface edge reproducibly.

Absolute values of the titanium and iron concentrations at the surface of the waveguide are determined by X-ray photoelectron spectroscopy (XPS). This method also provides information about the valence state $\left(\mathrm{Fe}^{2+} / \mathrm{Fe}^{3+}\right)$ of the in-diffused iron.

The penetration depths of titanium and iron in the $\mathrm{LiNbO}_{3}$ substrate are large compared to the thicknesses of the vacuum-deposited metal layers. Thus we expect [7] that the concentration profiles of both dopants can be described by Gaussian functions (diffusion from a finite source),

$$
c_{i}(y)=c_{i}^{0} \exp \left(-\frac{y^{2}}{2 \varrho_{i}^{2}}\right) ; \quad i=\mathrm{Ti}, \mathrm{Fe} .
$$

The penetration depth $\varrho$ depends on the temperature dependent diffusion constant $D$ and the diffusion time $t$,

$$
\varrho=\sqrt{2 D t} \text {. }
$$

We assume a local, linear relationship between the change of the extraordinary refractive index $\Delta n_{c}(y)$ and the concentrations $c_{i}(y)[7,8]$, and superimpose linearly the influences of titanium and iron ions,

$$
\Delta n_{\mathrm{c}}(y)=\xi_{\mathrm{Ti}} \frac{c_{\mathrm{Ti}}(y)}{c_{\mathrm{Ti}}^{0}}+\xi_{\mathrm{Fe}} \frac{c_{\mathrm{Fe}}(y)}{c_{\mathrm{Fe}}^{0}} .
$$

Then the parameters $\xi_{\mathrm{Ti}}, \xi_{\mathrm{Fe}}$ should be proportional to the absolute concentrations of titanium and iron, respectively.

\section{Experimental Results}

Using the microprobe the concentration profiles of titanium, iron, and niobium are measured in the waveguiding layer. The results for the relative concentrations enable a comparison to the refractive index profiles obtained by mode spectroscopy. Furthermore, it is possible to evaluate the diffusion constants of titanium and iron in $\mathrm{LiNbO}_{3}$.

In Fig. 1 the titanium concentration $c_{\mathrm{Ti}}$ is shown for the set of waveguides $\mathrm{Fe} 30$ to $\mathrm{Fe} 120$. The thickness of the deposited titanium layer $(80 \mathrm{~nm})$ is equal for all waveguides, while the thickness of the iron layer increases from $30 \mathrm{~nm}(\mathrm{Fe} 30)$ to $120 \mathrm{~nm}(\mathrm{Fe} 120)$. The diffusion times of titanium $(24+18 \mathrm{~h})$ and iron $(18 \mathrm{~h})$ and the annealing temperature $\left(1000{ }^{\circ} \mathrm{C}\right)$ are the same for all investigated waveguides. In addition, the reconstructed extraordinary 
refractive index profile $\Delta n_{\mathrm{e}}(y)$ of the waveguide $\mathrm{Fe} 0$ (80 $\mathrm{nm}$ titanium, annealed $24+18 \mathrm{~h}$ ) is included in the figure, too. This waveguide has the same concentration profile of titanium as the iron-doped samples indicating that the influence of the iron ions on the diffusion of titanium can be neglected.

In the region near to the surface the values of the titanium concentration deviate slightly for different waveguides, but no systematic dependence on the amount of in-diffused iron can be derived. The figure confirms a good correlation between the concentration profiles of titanium and the reconstructed refractive index profile.

By fitting Gaussian functions to the measured titanium profiles according to (1) we obtain an averaged penetration depth of $\bar{\varrho}_{\mathrm{Ti}}=(3.6 \pm 0.2) \mu \mathrm{m}$. With the diffusion time $t=42 \mathrm{~h}$ and (2) we evaluate the diffusion constant of titanium in $\mathrm{LiNbO}_{3}$ at $1000^{\circ} \mathrm{C}$ as $D_{\mathrm{Ti}}$ $=(4.4 \pm 0.5) \times 10^{-13} \mathrm{~cm}^{2} \mathrm{~s}^{-1}$.

At the surface the niobium concentration is reduced in all investigated waveguides by about $4 \%$ compared to the bulk value. The region of this decreased niobium concentration corresponds rather well to the penetration depth of the titanium ions. Furthermore, by $\mathrm{X}$-ray photoelectron spectroscopy we measure a titanium concentration of $3.3 \mathrm{~mol} \%$ (Fe80) at the surface.

The experimental values of the iron concentration $c_{\mathrm{Fe}}$ in the waveguiding layer are compared in Fig. 2. The iron concentration increases with increasing thickness of the deposited iron layer. The solid lines show the description of the dopant profiles by Gaussian functions. With the exception of the waveguide with the highest amount of in-diffused iron (Fe120) we obtain good agreement of measured values and fitted Gaussian curves. An explanation of this exception is proposed later. The given concentration values are (as well as the measurements of titanium concentrations) no absolute quantities, but describe the concentration relations of the waveguides Fe30 to Fe120. In the following the surface value

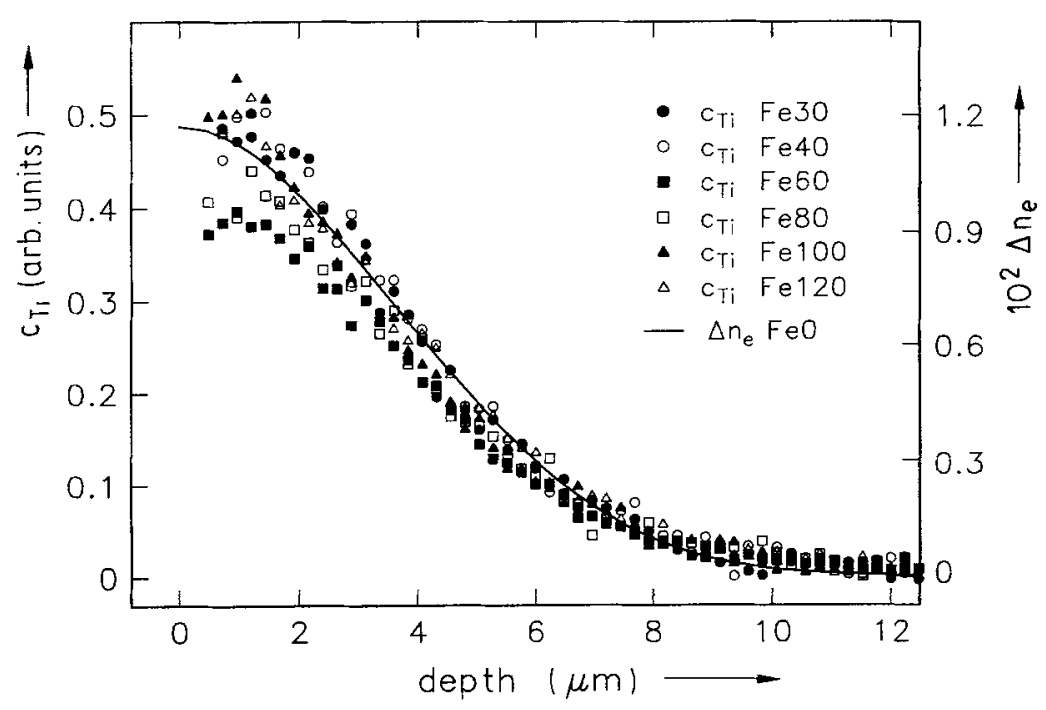

Fig. 1. Titanium concentration $c_{\mathrm{Ti}}$ in different titanium and iron in-diffused ( $\left.\mathrm{LNbO}_{3}: \mathrm{Ti}: \mathrm{Fe}\right)$ waveguides compared with the reconstructed refractive index profile $\Delta n_{\mathrm{e}}$ of the titanium in-diffused $\left(\mathrm{LiNbO}_{3}: \mathrm{Ti}\right)$ waveguide. The description of the waveguides corresponds to the thickness of the deposited iron layer. $\mathrm{FeO}$ is the titanium in-diffused waveguide without iron doping 


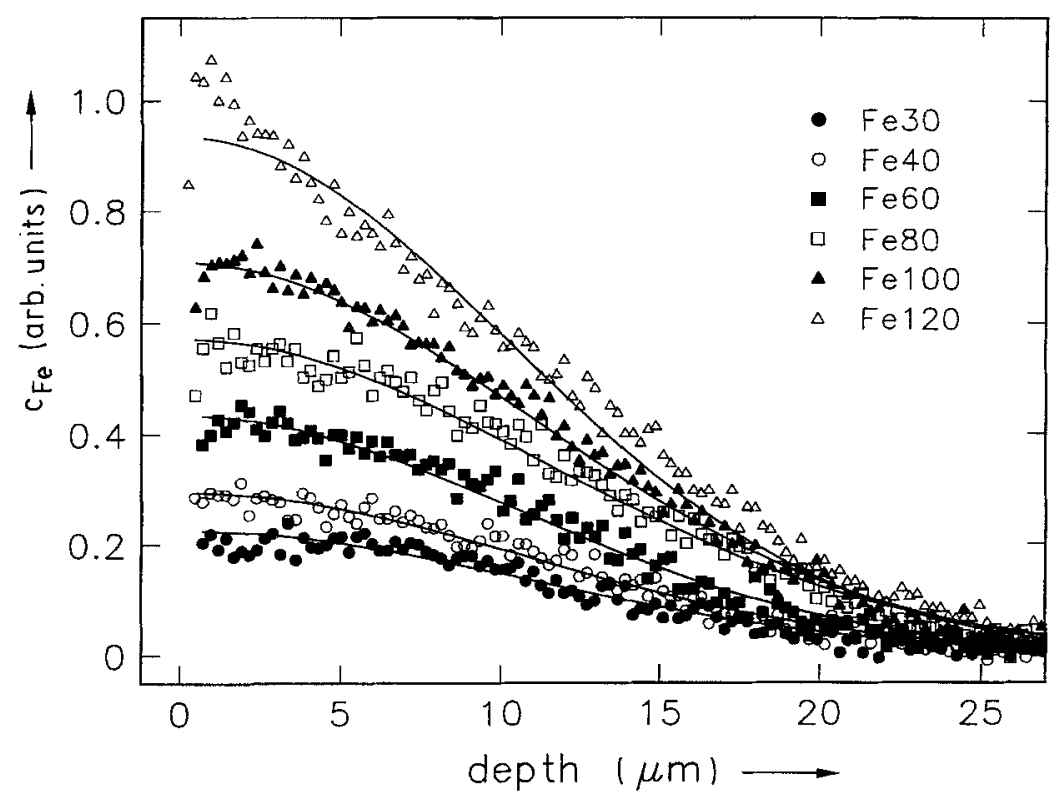

Fig. 2. Iron concentration $c_{\mathrm{Fe}}$ in different titanium and iron in-diffused $\left(\mathrm{LiNbO}_{3}: \mathrm{Ti}: \mathrm{Fe}\right)$ waveguides. The description of the waveguides corresponds to the thickness of the deposited iron layer. The solid lines are fits by Gaussian functions

$(x=0)$ of the Gaussian functions fitted to the concentration profiles is called the relative iron concentration $c_{\mathrm{Fe}}(x=0)$ of the waveguide.

The averaged penetration depth of the iron ions is $\bar{\varrho}_{\mathrm{Fe}}=(10.7 \pm 0.6) \mu \mathrm{m}$, considerably exceeding the value of titanium. Additionally, one has to take into account the shorter diffusion time of iron of $18 \mathrm{~h}$ (for comparison: titanium $42 \mathrm{~h}$ ). For the diffusion constant of iron in $\mathrm{LiNbO}_{3}$ at $1000{ }^{\circ} \mathrm{C}$ we obtain the value of $D_{\mathrm{Fe}}=(1.8 \pm 0.2) \times 10^{-11} \mathrm{~cm}^{2} \mathrm{~s}^{-1}$.

An absolute measurement of the iron concentration at the surface by X-ray photoelectron spectroscopy yields a value of $3.7 \mathrm{~mol} \%$ for the waveguide Fe80. Only $\mathrm{Fe}^{3+}$ is detected, while the concentration of $\mathrm{Fe}^{2+}$ is below the measuring accuracy.

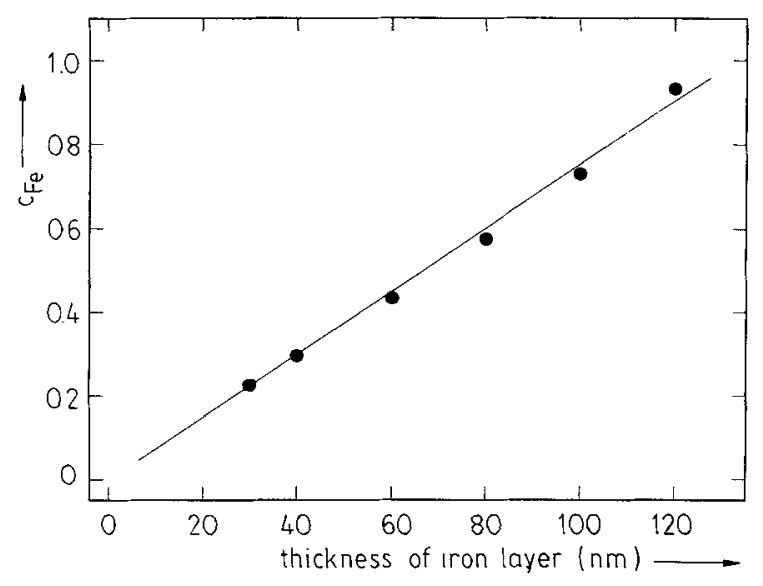

Fig. 3. Correlation of iron concentration $c_{\mathrm{Fe}}$ (relative values at the surface for the waveguides $\mathrm{Fe} 30$ to $\mathrm{Fel} 20$ ) with the thickness of the deposited iron layer. The solid line shows a linear regression of the two quantities 


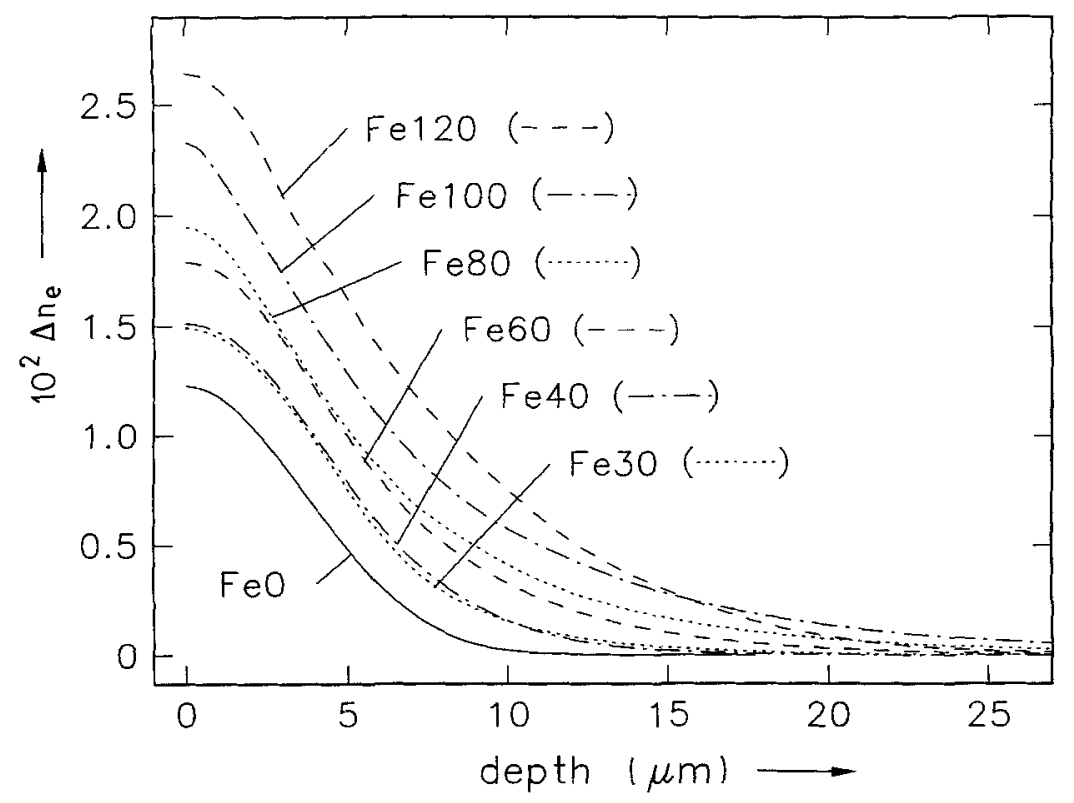

Fig. 4. Reconstructed refractive index profiles $\Delta n_{\mathrm{e}}$ (extraordinary polarisation) of the iron-doped $\left(\mathrm{LiNbO}_{3}: \mathrm{Ti}: \mathrm{Fe}\right)$ waveguides $(\mathrm{Fe} 30$ to $\mathrm{Fel} 20$ ) and the titanium in-diffused (LiNbO 3 : Ti) waveguide (FeO)

In Fig. 3 the relative iron concentration $c_{\mathrm{Fe}}(x=0)$ is correlated to the thickness of the deposited iron layers. The solid line elucidates the linear dependence of the two quantities. Obviously the in-diffused amount of iron does not reach the limit of its solubility in $\mathrm{LiNbO}_{3}$ even for the heavily doped samples.

The reconstructed extraordinary refractive index profiles of the waveguides $\mathrm{Fe} 0$ and $\mathrm{Fe} 30$ to Fe120 are shown in Fig. 4. The refractive index increases continuously with increasing iron concentration. A similar dependence is obtained for the ordinary refractive index profiles.

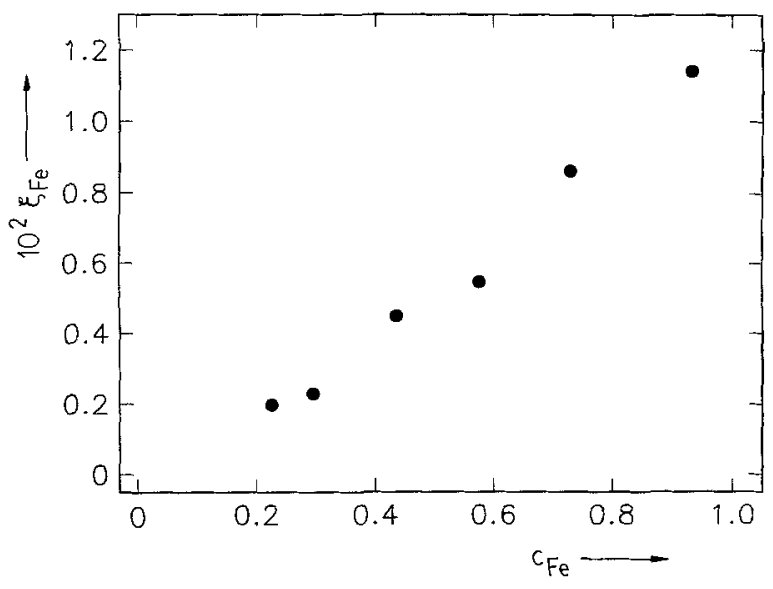

Fig. 5. Parameter $\xi_{\mathrm{Fe}}$ obtained by fitting of two superimposed Gaussian functions to the refractive index profiles $\Delta n_{\mathrm{e}}$ according to (3) as a function of the relative iron concentration $c_{\mathrm{Fe}}$ of the $\mathrm{LiNbO}_{3}: \mathrm{Ti}: \mathrm{Fe}$ waveguides $(\mathrm{Fe} 30$ to Fe120) 


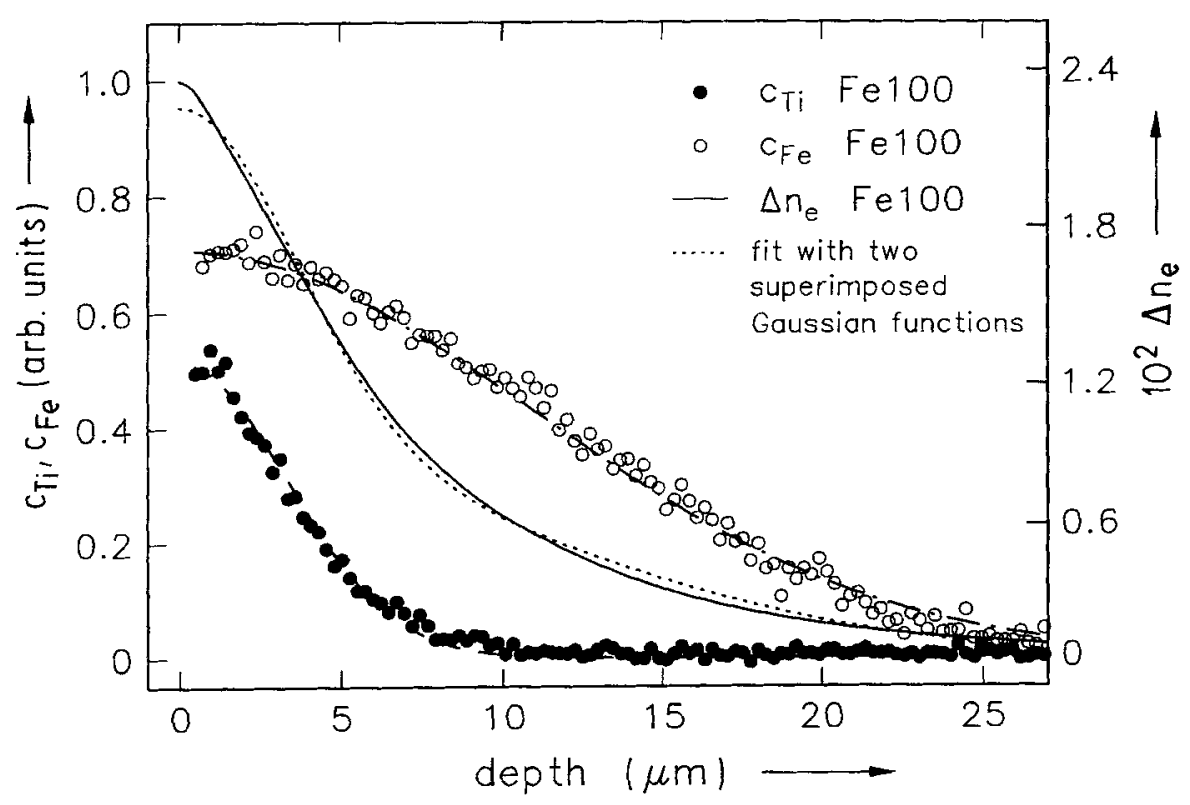

Fig. 6. Fit of the concentration profiles $c_{\mathrm{Ti}}, c_{\mathrm{Fe}}$, to the reconstructed extraordinary refractive index profile $\Delta n_{\mathrm{e}}$ for the waveguide Fe100. The dopant profiles $c_{\mathrm{T},}, c_{\mathrm{Fe}}$, are described by Gaussian functions (dashed lines, partly concealed). The parameters of the fit according to (3) are $\xi_{\mathrm{Ti}}=0.014$ and $\xi_{\mathrm{Fe}}=0.0086$

With the knowledge of the concentration profiles of titanium and iron in the waveguiding layer and the assumption of a local, linear dependence of the refractive index change $\Delta n_{\mathrm{c}}$ on the dopant concentrations $c_{i}$, it is possible to distinguish between the influence of titanium and iron ions on the extraordinary refractive index. The ordinary refractive index depends nonlinearly on the titanium concentration [6], and for this reason statements about the additionally iron-diffused samples are difficult. The extraordinary refractive index profiles, however, are correlated to the concentration profiles of titanium and iron according to (3). For this purpose we use the fitted Gaussian functions of Fig. 1 and 2.

The parameter $\xi_{\mathrm{Ti}}=0.014$ is obtained from the refractive index profile of the titanium in-diffused waveguide $\mathrm{Fe} 0\left(\xi_{\mathrm{Fe}}=0\right)$. With the assumption of equal titanium distributions in the waveguides ( $\mathrm{FeO}, \mathrm{Fe} 30$ to $\mathrm{Fe} 120$ ) this value is kept constant for the following fits to the extraordinary refractive index profiles of the iron-doped samples. Fig. 5 illustrates the obtained values of the parameter $\xi_{\mathrm{Fc}}$ as a function of the relative iron concentration. The measured dependence of the refractive index change on the iron concentration is practically linear.

As an example Fig. 6 shows the result of the comparison of the concentration profiles with $\xi_{\mathrm{Ti}}=0.014$ and $\xi_{\mathrm{Fe}}=0.0086$ and the reconstructed extraordinary refractive index profile $\Delta n_{\mathrm{e}}$ of the waveguide Fe100. The measured concentration profiles $c_{\mathrm{T}_{\mathrm{i}}}$ and $c_{\mathrm{Fe}}$ are fitted by Gaussian functions with a width of $\varrho_{\mathrm{Ti}}=3.3 \mu \mathrm{m}$ and $\varrho_{\mathrm{Fe}}=11.0 \mu \mathrm{m}$, respectively. The reconstructed refractive index profile is described very well by the fitted curve (superposition of the two Gaussian functions). For the other waveguides we obtain a similar good agreement. 


\section{Discussion}

The maximum values of the titanium concentration at the surface of the waveguides Fe 30 to Fe120 deviate by about $20 \%$ (Fig. 1), but a systematic dependence on the total concentration of in-diffused iron cannot be derived. Some $\mu \mathrm{m}$ away from the suface differences between the profiles of the waveguides are comparatively small. One possible reason for the deviations of the measured values at the surface is the non-perfect preparation of the polished endface for the microprobe analysis. Relative to this uncertainty, errors in the reproducability of the thickness of the deposited titanium layers, or slightly different annealing conditions of the waveguides are negligible.

The titanium profiles of the investigated iron-doped waveguides coincide with the refractive index profile of the waveguide $\mathrm{Fe} 0$ (only titanium in-diffused). The reduced niobium concentration near the surface of the waveguiding layer agrees well with the penetration depth of the titanium ions and is considerably different from the width of the iron profile. This indicates the incorporation of titanium ions on niobium sites. Quantitatively the value of niobium reduction at the surface of $4 \%$, measured with the microprobe, corresponds well to the titanium concentration of $3.3 \mathrm{~mol} \%$, obtained by X-ray photoelectron spectroscopy.

The measured concentration profiles of iron can be described by Gaussian functions (Fig. 2). Only the waveguide Fe120 is an exception. The concentration profile of this waveguide clearly shows deviations from a Gaussian lineshape near the surface and is more similar to an inverse error-function $\left(\operatorname{cerf}(x)=\int_{0}^{x} \exp \left(-t^{2} / 2\right) \mathrm{d} t\right)$. This function is a solution of the diffusion equation with an infinite source; thus it is expected for the profile shape of the in-diffused iron for thick deposited layers. The measured iron concentrations increase nearly linearly with increasing thickness of the iron layer (Fig. 3). A saturation of iron in $\mathrm{LiNbO}_{3}$ is not reached even for the highest concentrations of more than $5 \mathrm{~mol} \%$ (Fe120) iron relative to niobium (Fe80: $3.7 \mathrm{~mol} \%$ ). In homogeneously doped crystals, where iron oxide is already added to the melt, such high concentrations lead to problems: The crystals exhibit tensions, and they often crack when cooled to room temperature.

After annealing in an atmosphere of oxygen the waveguides are strongly oxidized; iron is mostly in the $\mathrm{Fe}^{3+}$ state. This is confirmed by X-ray photoelectron spectroscopy, measuring the $\mathrm{Fe}^{3+}$ concentration, with no detectable $\mathrm{Fe}^{2+}$. An estimation of the $\mathrm{Fe}^{2+}$ concentration yields a value which is at least two orders of magnitude lower than the concentration of $\mathrm{Fe}^{3+}$.

From the diffusion profiles of titanium and iron we deduce the corresponding diffusion constants. The obtained value of $D_{\mathrm{Ti}}=(4.4 \pm 0.5) \times 10^{-13} \mathrm{~cm}^{2} \mathrm{~s}^{-1}$ is in good agreement with earlier results $[7,9]$. The estimated error of this value results from the maximum deviation of the penetration depth $\varrho_{\mathrm{Ti}}$ around the averaged value $\bar{\varrho}_{\mathrm{Ti}}$. It should be noted that the diffusion constant of titanium depends decisively on the $\mathrm{Li} / \mathrm{Nb}$ ratio of the substrate and decreases from lithium-deficient $(48.1 \mathrm{~mol} \% \mathrm{Li})$ to lithium-rich $(50.0 \mathrm{~mol} \% \mathrm{Li})$ samples by one order of magnitude [10]. The calculated diffusion constant of iron in $\mathrm{LiNbO}_{3}$ is $D_{\mathrm{Fe}}=(1.8 \pm 0.2) \times 10^{-11} \mathrm{~cm}^{2} \mathrm{~s}^{-1}$. This value is 40 times larger than the one for titanium, while the radii of the two kinds of ions are nearly equal (Pauling radius, coordination number $6, \mathrm{Ti}^{4+}=61 \mathrm{pm}, \mathrm{Fe}^{3+}=65 \mathrm{pm}$ ). An explanation of the large differences may be different incorporation sites (iron on lithium site, titanium on niobium site) and the bonding energies of the two ions, respectively. 
The height of the ordinary and extraordinary refractive index profiles $\Delta n_{\mathrm{o}, \mathrm{c}}$ of the waveguides Fe30 to Fe120 increases with increasing amount of in-diffused iron (Fig. 4). We can separate the influence of titanium and iron on the extraordinary refractive index: the lineshape of the refractive index profile $\Delta n_{\mathrm{e}}(y)$ can be described by the concentration profiles of titanium and iron (Fig. 6). The iron ions lead to a linear increase of the extraordinary refractive index.

\section{Summary and Conclusions}

In this paper we examine the diffusion profiles of double-diffused $\mathrm{LiNbO}_{3}: \mathrm{Ti}: \mathrm{Fe}$ waveguides. These waveguides are interesting objects for the study of two- and four-wave mixing and efficient generation of phase-conjugate waves, respectively.

The concentration profiles of titanium, iron, and niobium are measured by an electron microprobe. The profiles of both titanium and iron can be described very well by Gaussian functions. At the surface the concentration of niobium is decreased, and the region of this reduction agrees well with the diffusion profile of titanium. Absolute concentration values of the two dopants at the surface are measured by X-ray photoelectron spectroscopy. Up to concentrations of more than $5 \mathrm{~mol} \%$ iron the limit of solubility of iron in $\mathrm{LiNbO}_{3}$ is not reached.

The extraordinary refractive index $\Delta n_{\mathrm{c}}(y)$ can be described by a linear superposition of the iron and titanium concentrations $c_{\mathrm{F}_{\mathrm{c}}}(y)$ and $c_{\mathrm{Ti}}(y)$ according to (3). This result allows the fabrication of waveguides with tailored properties.

\section{Acknowledgements}

Financial support of the Deutsche Forschungsgemeinschaft, SFB 225, is gratefully acknowledged. We thank T. Albers and M. Neumann for XPS measurements and P. Hertel for helpful discussions.

\section{References}

[1] R. S. WeIS and T. K. GaYlord, Appl. Phys. A 37, 191 (1985).

[2] A. Donaldonon, J. Phys. D 24, 785 (1991).

[3] V. E. Wood, P. J. Cressman, R. L. Holman, and C. M. Verber, Topics appl. Phys. 61, 45 (1989).

[4] D. Kip, R. Fink, T. Bartholomäus, and E. Krätzig, Optics Commun. 95, 33 (1993).

[5] D. KIP and E. KRÄTZIG, Optics Letters 17, 1563 (1992).

[6] P. Hertel and H. P. Menzler, Appl. Phys. B 44, 75 (1987).

[7] J. Vollmer, J. P. Nisius, P. Hertel, and E. KrätziG, Appl. Phys. A 32, 125 (1983).

[8] T. Brfmer, D. Kollewe, H. Koschmieder, and W. Heiland, Fresenius J. anal. Chem. 333, 485 (1989).

[9] M. Fukuma, J. Noda, and H. Iwasaki, J. appl. Phys. 49, 3693 (1978).

[10] R. J. Holmes and D. M. SMYTH, J. appl. Phys. 55, 3531 (1984). 